\title{
The relationship between outcome prediction and cognitive fatigue: A convergence of paradigms
}

\author{
G. R. Wylie ${ }^{1,2,3,4}$ - H. M. Genova $a^{2,4}$ J. DeLuca ${ }^{1,2,5}$ - E. Dobryakova ${ }^{2,4}$
}

Published online: 25 May 2017

(C) Psychonomic Society, Inc. 2017

\begin{abstract}
Cognitive fatigue is common after strenuous cognitive effort. A large body of literature has implicated a network of brain areas in fatigue, including the basal ganglia and cortical areas including ventro-medal prefrontal cortex and anterior cingulate cortex (ACC). Furthermore, the ACC has been shown to be involved in processes such as error and conflict monitoring, outcome prediction, and effort processing. Thus, the ACC appears to be one common denominator between clinical work on fatigue and research on outcome prediction and effort. In the present study, we examined whether the same region of the ACC is activated during the processing of errors and fatigue. Cognitive fatigue was induced by having subjects perform a difficult working memory task, during which they rated on-task fatigue. Activation associated with error processing was determined by using error trials on the working memory task. After localizing the region engaged in error processing, we evaluated whether there was a
\end{abstract}

Electronic supplementary material The online version of this article (doi:10.3758/s13415-017-0515-y) contains supplementary material, which is available to authorized users.

G. R. Wylie

gwylie@kesslerfoundation.org

1 Rocco Ortenzio Neuroimaging Center, Kessler Foundation, 1199 Pleasant Valley Way, West Orange, NJ, USA

2 Department of Physical Medicine and Rehabilitation, Rutgers University, Newark, NJ, USA

3 Department of Veterans' Affairs, War Related Illness and Injury Study Center, New Jersey Healthcare System, East Orange, NJ, USA

4 Neuropsychology and Neuroscience Research, Kessler Foundation, 120 Eagle Rock Ave, Suite 100, East Hanover, NJ 07936, USA

5 Department of Neurology, New Jersey Medical School, Rutgers University, Newark, NJ, USA relationship between BOLD activation of that region and ontask fatigue scores. The results showed that as subjects became more fatigued, they responded with longer latencies and increased accuracy for the more difficult task. Moreover, the ACC areas that were activated by error processing were also associated with fatigue. These results suggest that cognitive fatigue may be related to changes in effort and reward. We speculate that as the brain detects these changes, cognitive fatigue is generated as a way for the brain to signal itself that the effort required for the task no longer merits the rewards received for performing it.

Keywords Cognitive control $\cdot$ Reward $\cdot$ Cognitive fatigue Outcome prediction

Mental or cognitive fatigue is a common experience. We experience cognitive fatigue after doing mental work rather than physical work, and it is common after a day at the office, after preparing your taxes, or after driving for a long time (Rothengatter \& Huguenin, 2004). This last example is illustrative of several important aspects of cognitive fatigue. After the sustained effort of having driven for long enough to become fatigued, we feel we have expended many of our cognitive resources (indeed, we feel "spent"), and it is not always easy to continue. Indeed, we often feel that effort is required to continue our journey, and when we reach this fatigued state, it is common to slow down and to be more cautious than when we start the journey. That is, we trade off speed for accuracy (Rozand, Lebon, Papaxanthis, \& Lepers, 2015) - choosing to travel at a slower rate to achieve greater accuracy (Rothengatter \& Huguenin, 2004).

Although cognitive fatigue is a common experience to some degree, it disproportionately affects individuals who have sustained a neural injury (e.g., traumatic brain injury or stroke) 
or who have a disease of the central nervous system (e.g., multiple sclerosis). Whereas most people feel cognitively fatigued on occasion, individuals with brain injury or disease experience cognitive fatigue nearly all the time, and in previous work we have speculated that fatigue results from an imbalance between effort and reward (Dobryakova, DeLuca, Genova, \& Wylie, 2013; Dobryakova, Genova, DeLuca, \& Wylie, 2015).

In clinical populations, the effort-reward imbalance may be due to damage or dysfunction of the basal ganglia (Chaudhuri \& Behan, 2000; DeLuca, Genova, Hillary, \& Wylie, 2008; Dobryakova et al., 2015; Genova et al., 2013; Kohl, Wylie, Genova, Hillary, \& Deluca, 2009). In healthy samples, it has been shown that subjects are able to overcome their fatigue if they are motivated by rewarding outcomes (Boksem, Meijman, \& Lorist, 2006; Lorist et al., 2009). For example, Lorist et al. induced fatigue by having subjects perform a mentally demanding task for two hours. During this time, fatigue ratings increased and performance deteriorated (longer response times [RTs] and more errors). However, at the end of the 2-h session, when subjects were offered a monetary reward to perform better, they were able to improve their performance, suggesting that when motivated, subjects were able to increase their effort on the task and overcome their fatigue.

One way to interpret these results is that when subjects perform a task, they expend cognitive resources. After a sufficiently long period of repetitive task performance, the cognitive resources they have available begin to diminish, and effort is required to continue to perform the task. If the reward they receive for continued task performance does not increase, then there is a shift in the payoff matrix: The effort required increases, but the reward received does not. This shift in the payoff matrix may be experienced as fatigue. On the other hand, fatigue can be overcome if the payoff matrix is altered such that continued performance results in larger rewards (Boksem et al., 2006; Lorist et al., 2009). Put another way, after repetitive task performance, a discrepancy develops between the reward subjects expect to receive and the effort they are required to expend.

A large body of literature has implicated the cortico-striatal network in fatigue, with the striatum, the ventromedial prefrontal cortex (vmPFC), and the anterior cingulate cortex (ACC) playing key roles. Indeed, neuroimaging studies investigating fatigue have often reported the involvement of the ACC (DeLuca et al., 2008; Genova et al., 2013; Pardini, Bonzano, Mancardi, \& Roccatagliata, 2010; Pardini, Krueger, Raymont, \& Grafman, 2010). Importantly, a separate body of literature implicates the same network in effort and reward processing. The vmPFC and ACC, in particular, have been associated with several cognitive domains, including error monitoring (e.g., Brown \& Braver, 2005), conflict monitoring (e.g., Botvinick, Nystrom, Fissell, Carter, \& Cohen, 1999), and outcome prediction (Alexander \& Brown, 2010; Zarr \& Brown, 2016). For example, when a subject makes an error, there is a discrepancy between the expected outcome (a correct response) and the actual outcome (the erroneous response; Niv \& Schoenbaum, 2008; Schultz, 2016). This discrepancy seems to be registered in the ACC, as demonstrated by a robust BOLD response in functional neuroimaging studies (Engström, Karlsson, Landtblom, \& Craig, 2015), as well as an error-related negativity (ERN) in electrophysiological studies (Yeung, Botvinick, \& Cohen, 2004). Indeed, the ACC has been found to respond to errors even when subjects are unaware of having responded erroneously (Hester, Foxe, Molholm, Shpaner, \& Garavan, 2005).

Thus, the literature on cognitive fatigue and a separate literature on outcome prediction both report brain activation in the ACC. Moreover, it has been hypothesized that fatigue may be related to an imbalance between effort and reward (i.e., a type of outcome prediction; Boksem \& Tops, 2008; Dobryakova et al., 2013). In the present study, we tested whether precisely the same regions of the ACC are activated during error processing and as cognitive fatigue increases during task performance. To do this, we induced cognitive fatigue by having subjects perform four blocks of a difficult working memory task and four blocks of a control task. Before and after each of these blocks, we asked subjects to rate their level of "state," or on-task, fatigue, on a scale from 0 (no fatigue) to 100 (the most fatigue they had experienced).

We assessed activation associated with error processing by using the error trials of the difficult working memory task as a localizer task (i.e., a task used to localize the brain areas associated with error processing). The task was sufficiently difficult that errors were not uncommon, and this allowed us to establish the area in the ACC associated with error processing by comparing activation during error trials to activation during correct trials. Having localized a large area associated with error processing in the ACC, we then assessed whether this brain area was sensitive to fatigue by testing whether activation on the two tasks correlated with the fatigue ratings in this area of the ACC. To ensure that the resulting correlations were not contaminated by the large activation on error trials, only correct trials of the two tasks were included in the second analysis. Our hypothesis was that some of the same areas of the ACC that were active during error processing would also be related to fatigue, since both error processing and fatigue may involve outcome predictions.

\section{Method}

Subjects Twenty-three healthy, right-handed subjects (14 women, nine men) took part in this study. These subjects were recruited as healthy controls in a larger study investigating fatigue in multiple sclerosis and traumatic brain injury. Their mean age was 41.7 years $(S D=12.4)$, and all were native English speakers. All subjects were screened to ensure that it 
was safe for them to have a functional magnetic resonance imaging (fMRI) scan. They were also screened to ensure that they did not have any psychiatric conditions (e.g., schizophrenia), neurological disease (e.g., multiple sclerosis), or neurological insult (e.g., traumatic brain injury) that would affect brain function. Each was paid $\$ 100$ for participation, and all provided informed consent, in accordance with the Institutional Review Boards at the Kessler Foundation and Rutgers University Medical School.

Neuroimaging acquisition A 3-T Siemens Allegra scanner was used to acquire all neuroimaging data. A T2*-weighted pulse sequence was used to collect functional images in 32 contiguous slices during eight blocks (four at each of two difficulty levels; see below), resulting in 140 acquisitions per block (echo time $[\mathrm{TE}]=30 \mathrm{~ms}$, repetition time $[\mathrm{TR}]=2,000$ $\mathrm{ms}$, field of view [FOV] $=22 \mathrm{~cm}$, flip angle $=80^{\circ}$, slice thickness $=4 \mathrm{~mm}$, matrix $=64 \times 64$, in-plane resolution $=$ $3.438 \mathrm{~mm}^{2}$ ). A high-resolution magnetization-prepared rapid gradient echo (MPRAGE) image was also acquired (TE $=$ $4.38 \mathrm{~ms}, \mathrm{TR}=2,000 \mathrm{~ms}, \mathrm{FOV}=220 \mathrm{~mm}$, flip angle $=8^{\circ}$, slice thickness $=1 \mathrm{~mm}, \mathrm{NEX}=1$, matrix $=256 \times 256$, inplane resolution $=0.859 \times 0.859 \mathrm{~mm}$ ), which was used to normalize the functional data into standard (Talairach) space (Talairach \& Tournoux 1988).

Behavioral paradigm and data Behavioral data acquisition and stimulus presentation were administered using the EPrime software package (Schneider, Eschman, \& Zuccolotto, 2002). During the fMRI scan, subjects were presented with the $n$-back working memory task, in which task difficulty was varied by presenting the zero-back condition, which places a low load on working memory, and the two-back condition, which places a higher load on working memory. We presented four blocks of each level of the $n$-back task (eight blocks total), with 65 trials per block. The four blocks of each task were always presented together (i.e., the two tasks were not interleaved), and the order of presentation (zero-back first vs. two-back first) was counterbalanced across subjects. During the zero-back task (control task), subjects were asked to respond each time the target letter " $\mathrm{K}$ " was presented on the screen, and during the two-back task, subjects were asked to respond when the target letter corresponded to the letter presented two trials before (e.g., R N Q N . . .). Letters were presented in white (Arial 72-point font) on a black background. Of the 26 letters in the English alphabet, nine were excluded in order to enhance the discriminability of the letters used as stimuli. The following letters were used (with equal frequencies): A B C D F H J K M N P Q R S T V Z. The letter stimuli remained on the screen for $1.5 \mathrm{~s}$, followed by a $500-\mathrm{ms}$ intertrial interval (ITI), and the time between successive trials was jittered to allow for the data to be deconvolved as an event-related design. The jittering was optimized using the
Optseq2 program (https://surfer.nmr.mgh.harvard.edu/ optseq/). The jittering was achieved by inserting between zero and six null events between successive trials. The duration of each null event was a multiple of the length of the trial (in this case, $2 \mathrm{~s}$ ), drawn from a distribution following a power function. The majority of intertrial intervals were $500 \mathrm{~ms}$ (zero null events), followed by $2 \mathrm{~s}$ (one null event), and so on. The average intertrial interval was $1,587.87 \mathrm{~ms}$, and the standard deviation was $1,769.7$ ms. All subjects practiced both tasks prior to the scanning session.

To ensure comparable stimulation across subjects, the stimuli always remained on the screen for $1.5 \mathrm{~s}$ (i.e., they were not removed when subjects responded), and each run lasted the same amount of time $(260 \mathrm{~s})$. The average amount of time between successive blocks was $2 \min 4 \mathrm{~s},(S D=2 \min 17 \mathrm{~s})$.

Visual analog scale (VAS) To evaluate the level of on-task or "state" fatigue, subjects were presented with a VAS before and after each block of the $n$-back task. Subjects were asked, "How mentally fatigued are you right now?" and to indicate their level of fatigue on a scale from 0 to 100 , with 0 being not fatigued at all and 100 being extremely fatigued. To mask the purpose of the study, five additional VASs were administered as well, in randomized order. These assessed happiness, sadness, pain, tension, and anger.

fMRI data processing For each of the fMRI blocks, the first five images were discarded to ensure steady-state magnetization. All images were preprocessed using the Analysis of Functional NeuroImages software (AFNI, version AFNI_16.0.00; Cox 1996). The realignment, co-registration, and normalization were done in a single transform. This was accomplished by calculating and saving the parameters necessary for realignment using 3 dvolreg (i.e., the spatial co-registration of all images in each time series to the first image of the series). Next, the parameters necessary to co-register the first image in each time series with the high-resolution MPRAGE were calculated and saved (using 3dAllineate). Third, the MPRAGE image $(1 \times 1 \times 1 \mathrm{~mm}$ voxels $)$ was warped into standard space using a nonlinear warping algorithm (3dQwarp), and the warping parameters were saved. Finally, the transforms necessary to realign, co-register, and warp the data into standard space were combined and applied to the functional time series data in a single transformation. Time series with excessive motion (translations $>3.5 \mathrm{~mm}$ or rotations $>1.5$ ) were flagged and excluded from the group-level analyses. The images were then smoothed using an $8 \times 8 \times 8 \mathrm{~mm}$ Gaussian smoothing kernel (using 3dBlurToFWHM) and scaled to the mean intensity (using 3dcalc). Each of the four blocks of each task (zero-back and two-back) was then deconvolved separately (using 3dDeconvolve), with correct and error trials being coded as separate regressors. Motion parameters and two polynomial 
regressors (to model signal drift) were included as regressors of no interest.

Data analysis For the neuroimaging data, the analysis was conducted in two steps. The first step was designed to identify regions in the ACC that were associated with error processing by comparing the BOLD responses on error and correct trials, using a $t$ test (whole-brain analysis). Because subjects made very few errors during the zero-back task (accuracy was $99 \%$ ), only the two-back task was used for this purpose. On the basis of these results, a mask was created of the area in the ACC that was more active on error than on correct trials (number of voxels $=2,188$; location of peak activation in $x$-, $y$-, $z$ coordinates $=5,21,64$ ), and this was used to constrain the second step of the analysis.

In the second step, the data from only the correct trials from both the zero-back and two-back tasks were used. These data were analyzed with three linear mixed-effects analyses, using the R statistical package. For all three analyses, the factors were task (zero-back and two-back) and run (Run 1-4), with subject as a random factor.

In the first model, the VAS fatigue scores that were acquired before and after each block were averaged to estimate the fatigue during each block, and these average scores were entered as a quantitative variable. We refer to this model as "Fatigue_average." In the second model, the same average VAS fatigue scores were used, but the difference between the VAS fatigue scores before and after each block was entered as an additional quantitative variable. We refer to this model as "Fatigue_average + difference." In the third model, the VAS fatigue scores recorded before each run were entered as one quantitative variable, and the VAS fatigue scores after each run were entered as a second quantitative variable. We refer to this model as "Fatigue_preVpost." Supplementary Fig. 1 shows a plot of the VAS fatigue scores. These analyses allowed us to identify voxels that were responsive to different aspects of subjects' state fatigue ratings. For all analyses, each subject's data contributed equally to the results.

The response time (RT) and accuracy data were also analyzed with the same three linear mixed-effects analyses. This allowed us to assess the effects of task and run, and to examine whether different aspects of fatigue correlated with either RT or accuracy (and if any such correlation was modified by task or run).

Correction for multiple comparisons For the $\mathrm{MMI}$ data, all group-level statistical maps were thresholded using both the alpha level and the cluster size correction (i.e., the extent of activation). For the first step in the fMRI data analysis, in which voxels sensitive to error processing were identified, the alpha level was set at $p<.01$ and the cluster size was set at 289 contiguous voxels (voxel size $=2 \times 2 \times 2 \mathrm{~mm}$ ). The results of Monte Carlo simulations showed that this combination resulted in a corrected alpha level of $p<.05$. For the second step, in which only the area of activation in the ACC was interrogated, Monte Carlo simulations showed that the cluster size correction should be set at 47 voxels. Monte Carlo simulations were conducted using 3dClustSim (compile date Jan 1, 2016).

\section{Results}

The first two models (Fatigue_average and Fatigue_average + difference) produced the same pattern of results. Moreover, in the Fatigue_average + difference model, there was no effect of the difference in fatigue before and after the block, nor did this variable interact with any other factor. This was the case in the analyses of the behavioral data as well as the fMRI data. We therefore do not present the results from the Fatigue average + difference model.

\section{Fatigue_average: Behavioral data}

In the RT data, as expected, we observed a main effect of task $[F(1,146.68)=181.56, p<.0001]$ that resulted from subjects responding with longer latencies when performing the twoback task (mean $\pm S D=742.58 \pm 111.82$ ) than when performing the zero-back task $(583.64 \pm 93.52)$ (see Fig. 1). The only other reliable effect was the effect of fatigue on RTs $[F(1,104.1)=7.5, p<.01]$ (see Fig. 1$)$, which had a positive slope (coefficient) of 2.67 (i.e., for every unit increase in fatigue, subjects responded $2.67 \mathrm{~ms}$ slower). The coefficients for this model are shown in Supplementary Fig. 2.

In the accuracy data, we also found a main effect of task $[F(1,146.67)=80.33, p<.0001]$ that resulted from subjects making more errors when performing the two-back task $(9.0 \%$ $\pm 10 \%)$ than when performing the zero-back task $(1.0 \% \pm 3 \%)$ (see Fig. 1). The interaction between task and fatigue $[F(1$, 148.32 ) $=7.23, p<.01$ ] was also significant (see Fig. 1). This interaction resulted from a negative relationship between fatigue and accuracy for the zero-back task (slope/coefficient = $-0.0007)$ and a positive relationship for the two-back task (slope/coefficient $=0.0004)$. The coefficients for this model are shown in Supplementary Fig. 2.

\section{Fatigue_average: Neuroimaging data}

Error processing To ensure that there were sufficiently many error trials to analyze, we first calculated the number of incorrect trials on the two-back task for each subject: The numbers of incorrect trials ranged from five to 30 (mean $=16.2, S D=$ 8.0 ), giving us confidence in the signal associated with these error trials. For the imaging results associated with error processing, we found a robust difference between incorrect and correct trials (see Fig. 2 and Table 1). The largest difference between incorrect and correct trials was found in the ACC, extending from the supplementary motor area (SMA) down to 


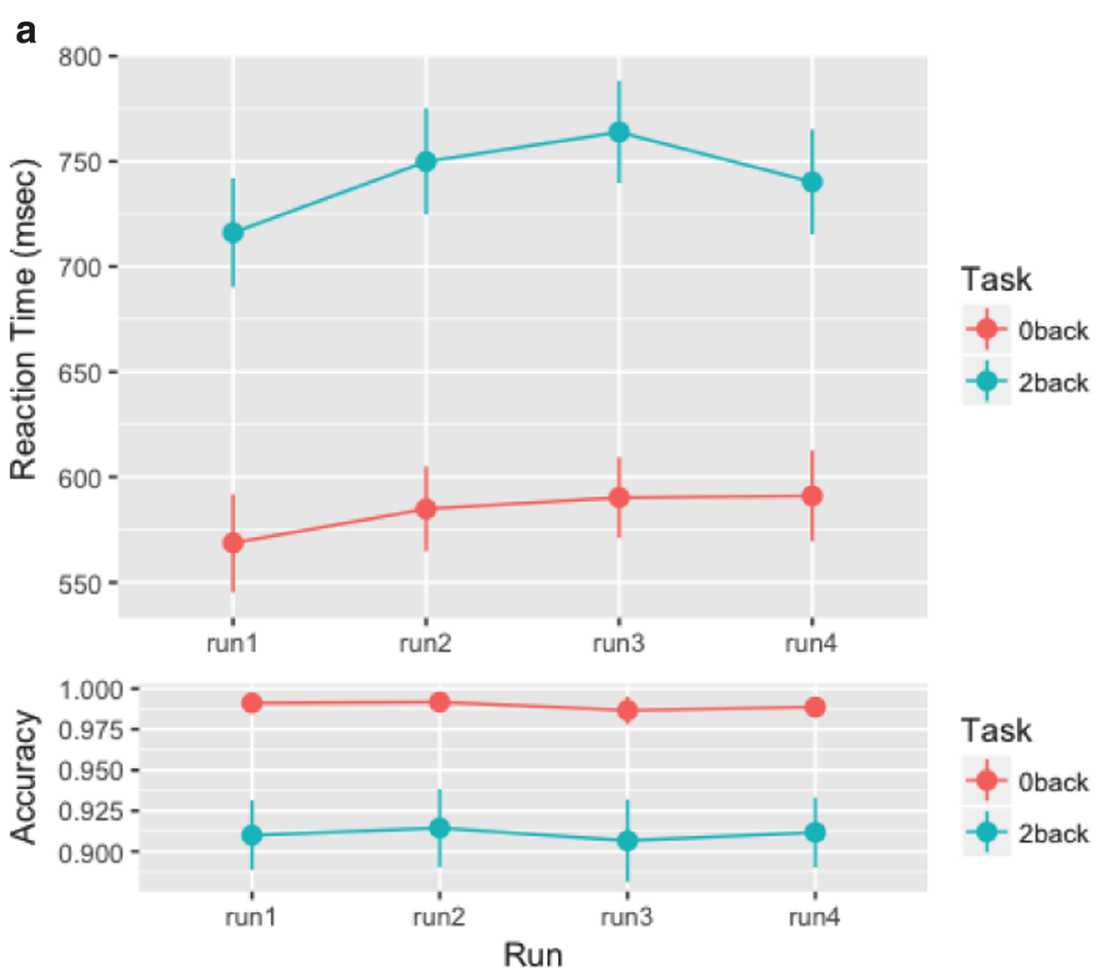

b RT: Task $x$ Average Fatigue

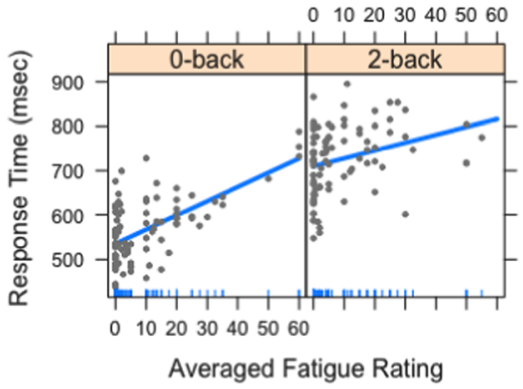

C RT: Fatigue_pre $x$ Task

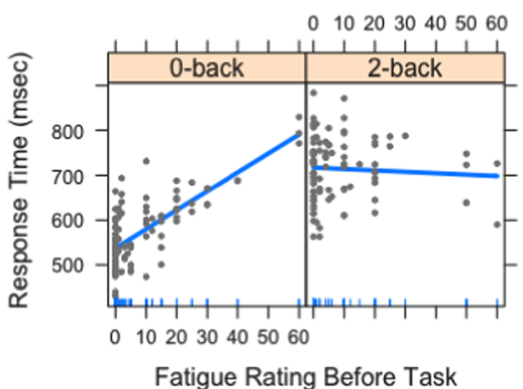

Fig. 1 Behavioral data and their interaction with fatigue. (a) Average reaction times (RTs) and accuracy for the zero-back task and the twoback task, separated by run. Error bars represent the standard errors of the means. (b) The (nonsignificant) Task $\times$ Average Fatigue interaction in

the cingulate cortex, and forward to the ACC (see Fig. 2). Additionally, we observed bilateral activation of the insula that was greater during error trials than during correct trials (see Fig. 2 and Table 1).

\section{Accuracy: Task $\times$ Average Fatigue}

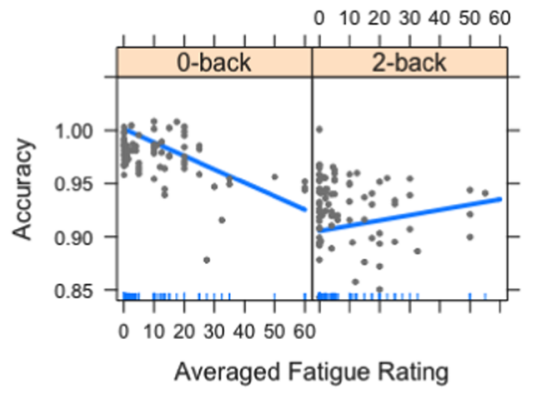

RT: Task x Fatigue_post

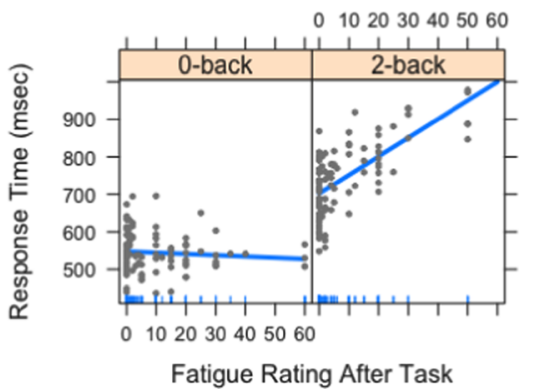

RTs (left). For accuracy (right), this interaction was significant. (c) Interactions between Fatigue pre and task (left) and Fatigue post and task (right), both in RTs

Fatigue processing Here we analyzed only the correct trials from the zero-back and two-back tasks, using subjects' averaged ratings of fatigue as a quantitative variable in the analysis. Furthermore, this analysis was restricted to the 


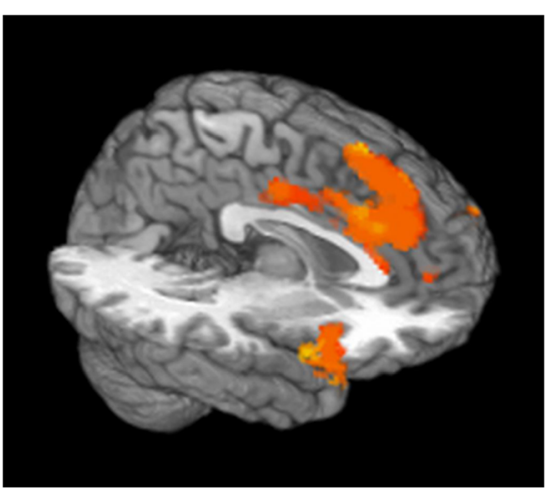

Fig. 2 Error-related activation (incorrect $>$ correct). Orange areas show those areas that were more active on incorrect than on correct trials. Two regions are depicted here: anterior cingulate cortex and the insula. All regions are thresholded at $p<.05$, corrected

area in the ACC that was associated with error processing. This analysis revealed two areas where average fatigue interacted with task (see Fig. 3 and Table 2) (there was no main effect of average fatigue in any area). In both, activation increased as average fatigue increased, but only for the two-back task (for the zero-back task, activation decreased as average fatigue increased). For the more anterior region in the ACC (shown in Fig. 3), there was a positive relationship (slope) between average fatigue and activation during the two-back task (0.003); however, the relationship (slope) was negative for the zero-back task (0.002). For the more posterior region in the cingulate cortex, the pattern was similar to that seen in the ACC, though less strong: a positive relationship between average fatigue and activation during the two-back task (0.001), and a negative relationship between average fatigue and zero-back task activation $(-0.001)$. To ensure that the data contained as little error-related variability as possible, this analysis was rerun excluding not only trials on which subjects had made errors, but also trials following an error. The pattern of results was unchanged, though the clusters of activation (see Fig. 3 and Table 2) were moderately larger (the ACC cluster was 207 voxels [rather than 161]; the cingulate cluster was 117 voxels [rather than 96]). For the sake of completeness, we also show the main effect of task in Supplementary Fig. 5, and report all the areas where average fatigue interacted with task in Supplementary Table 1.

\section{Fatigue preVpost: Behavioral data}

For this analysis the fatigue ratings collected before ("Fatigue pre") and after ("Fatigue post") each block were included as separate quantitative variables in the model. In the analysis of RTs, we (again) found a main effect of task $[F(1$, $152.9)=132.9, p<.0001]$, resulting from subjects responding with longer latencies on the two-back task than on the zeroback task. There were also significant effects of Fatigue_pre $[F(1,168.1)=3.9, p<.05]$ and Fatigue post $[F(1,151.8)=$ $4.9, p<.05]$ on RTs, and both Fatigue pre and Fatigue_post interacted with task (see Fig. 1). The interaction between task and Fatigue pre $[F(1,154.8)=5.1, p<.05]$ resulted from a positive relationship between the fatigue that subjects reported prior to the zero-back task and the RTs on that task: More fatigue prior to blocks of the zero-back task was associated with longer RTs during those blocks. This was not the case for the two-back task, in which the relationship was very weakly negative. The interaction between task and Fatigue post $[F(1$, $151.7)=9.1, p<.01]$ resulted from a positive relationship between RTs on the two-back task and the fatigue that subjects reported following two-back task performance: Longer RTs during two-back blocks were associated with more fatigue after those blocks. This was not the case for the zero-back task, in which the relationship was weakly negative (see also Supplementary Fig. 4 for a plot of the coefficients of the model).

In the analysis of accuracy, the only effect was that of task $[F(1,155.1)=76.3, p<.0001]$, which (as before) resulted from subjects making fewer errors during the zero-back task than during the two-back task.

\section{Fatigue_preVpost: Neuroimaging data, fatigue processing}

When Fatigue pre and Fatigue post were included as separate quantitative variables in the group-level analysis, restricted to the regions of interest associated with error processing, there was one area where task interacted with Fatigue post (see Table 2). This was in the pre-SMA, and as Fig. 3 shows, the interaction in the BOLD data was similar to that seen in the analysis of average fatigue as well as in the RT data. For the two-back task, there was a positive relationship between BOLD activation and fatigue scores after each block: More BOLD activation during the two-back blocks was associated

Table 1 Areas of reliable brain activation for the analysis of error trials during the two-back task

\begin{tabular}{|c|c|c|c|c|c|c|}
\hline Error Processing in the Two-Back Task & BA & $x$ & $y$ & $z$ & Voxels & $t$ Stat. \\
\hline Medial prefrontal cortex/Anterior cingulate cortex/ Supplementary motor area & $6 / 10 / 32$ & 5 & 21 & 64 & 2,354 & 2.89 \\
\hline Insula/Inferior frontal gyrus & 45 & 57 & 15 & 0 & 727 & 3.02 \\
\hline Insula/Inferior frontal gyrus & 47 & -49 & 15 & -2 & 620 & 3.04 \\
\hline
\end{tabular}

"BA" denotes Brodmann areas; " $x$," " $y$," and " $z$ " denote the location of the voxel in each cluster with the highest activation; "Voxels" denotes the number of voxels in each cluster; " $t$ Stat." denotes the $t$ statistic associated with the voxel of highest activation. 


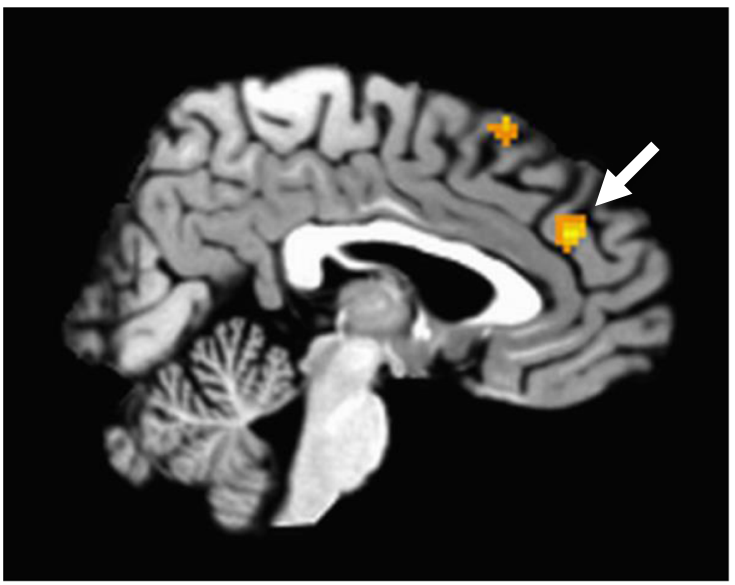

\section{Task X Average Fatigue [ACC]}

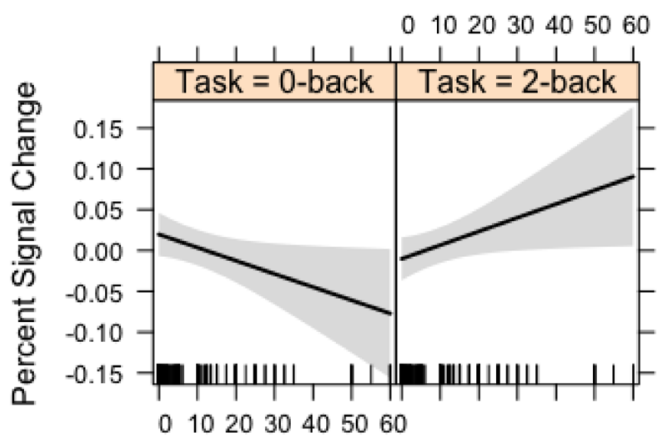

Averaged Fatigue Rating

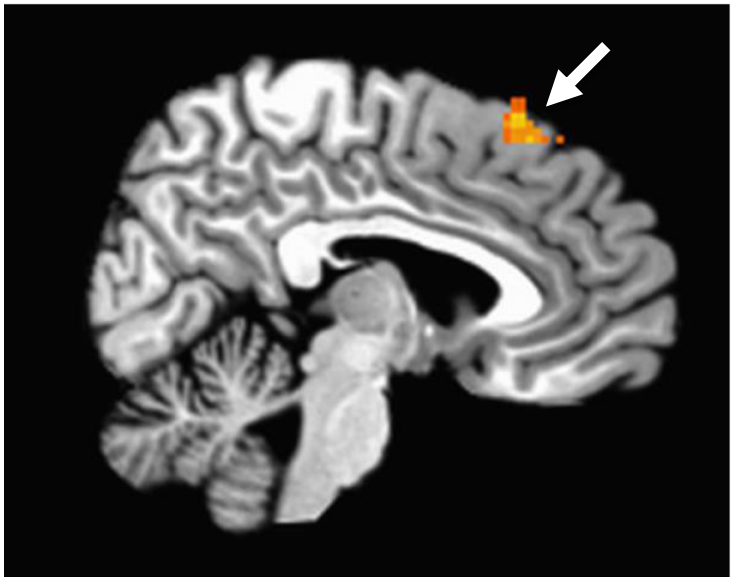

\section{Task X Fatigue_post [Cingulate Cortex]}

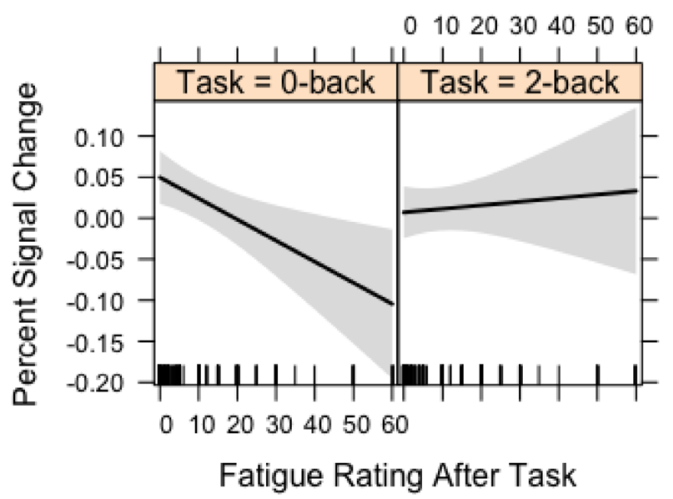

Fig. 3 Relationship between fatigue and activation. The top panels show the relationship between average fatigue and activation in the cingulate cortex and in the anterior cingulate cortex (ACC). In the left panel, orange areas show areas where fatigue interacted with task. In the right panel, the interaction is shown for the larger, more anterior area of activation

with more fatigue following those blocks. For the zero-back task, the relationship was negative: Less BOLD activation during the zero-back blocks was associated with more fatigue following those blocks.

Relationship between neural response and behavior To investigate whether the activation in the ACC was related to behavioral performance, we ran four additional analyses. For both RTs and accuracy, we ran a linear mixed-effects model

(indicated in the left panel by an arrow). The bottom panels show the relationship between Fatigue post and activation in the cingulate cortex. On the left, the activated area in the pre-SMA is indicated by an arrow; on the right, the interaction is shown. All regions are thresholded at $p<.05$, corrected

using the percent of signal change in each of the two clusters identified in the Fatigue_average analysis as a quantitative variable (resulting in four analyses). Because these analyses were conducted to investigate the relationship between brain activation and behavior, only interactions involving brain activation and behavior were of interest.

For the analysis of RTs, we observed a significant interaction between task and activation in the ACC cluster $[F(1$, $160.62)=3.80, p=.05]$. This can be seen in Fig. 4. For the

Table 2 Areas of reliable brain activation for the interaction between fatigue and task

\begin{tabular}{|c|c|c|c|c|c|c|}
\hline Fatigue $\times$ Task Interaction & BA & $x$ & $y$ & $z$ & Voxels & $F$ Stat. \\
\hline \multicolumn{7}{|l|}{ Average Fatigue $\times$ Task } \\
\hline Anterior cingulate cortex & 32 & 3 & 39 & 26 & 161 & 16.94 \\
\hline Supplementary motor area \& Cingulate cortex & $32 / 8$ & 11 & 23 & 46 & 96 & 14.51 \\
\hline \multicolumn{7}{|l|}{ Fatigue_post $\times$ Task } \\
\hline Supplementary motor area & $6 / 8$ & 7 & 23 & 56 & 117 & 24.22 \\
\hline
\end{tabular}

"BA" denotes Brodmann areas; " $x$, , " $y$," and " $z$ " denote the location of the voxel in each cluster with the highest activation; "Voxels" denotes the number of voxels in each cluster; " $F$ Stat." denotes the $F$ statistic associated with the voxel of highest activation. 
two-back task, there was a negative correlation between RT and brain activation, whereas for the zero-back task, there was essentially no relationship between brain activation and RT. For the cingulate cluster, no interactions emerged between RT and activation.

For the analysis of accuracy, there was also a significant interaction between task and activation in the ACC cluster $[F(1,163.04)=6.68, p<.05]$. As Fig. 4 shows, for the twoback task we observed a positive correlation between accuracy and brain activation, whereas the zero-back task showed essentially no relationship between brain activation and accuracy. For the cingulate cluster, the interaction between RT and activation was not significant. Taken together, these results show that increasing brain activation in the ACC was associated with faster, more accurate performance during the twoback task, but not during the zero-back task.

Potential confounds One potential alternative explanation for these data is that the ACC was not responding to fatigue but rather to task engagement. For example, subjects may have been more engaged during the more difficult two-back task than during the less difficult zero-back task. To test this, we performed two additional analyses on the BOLD data. In the first, we included the average accuracy during each run in addition to the average VAS score for each run in the group-level analysis, on the hypothesis that when subjects were more engaged they would make fewer errors. The pattern of results remained unchanged, though the numbers of voxels in the two clusters of activation differed (ACC cluster, 85 voxels; cingulate cluster, 49 voxels). In the second, we reran the deconvolution on each subject's data, including the number of trials since an error as a regressor of no interest, to test the hypothesis that when subjects were more engaged they would have longer runs of trials between errors. Again, the pattern of results remained unchanged, though in this case there was a marked decrease in the number of voxels in each cluster (ACC cluster, 25 voxels; cingulate cluster, 36 voxels). This change in cluster size probably resulted from collinearities in the model between the number of trials since an error and the regressors included to model signal drift. Because subjects made very few errors in either task, but particularly for the zero-back task, the regressor for trials since an error was a monotonically increasing function, and was therefore highly correlated with the linear regressor included in the model to account for signal drift.

Because we found a relationship between RT and fatigue, and also because RT has been shown to affect the BOLD signal in the ACC (Carp, Fitzgerald, Taylor, \& Weissman, 2012; Grinband et al. 2011; Mohamed et al. 2004), we reran the deconvolution on each subject's data, including RT as a regressor of no interest. Again, the pattern of results
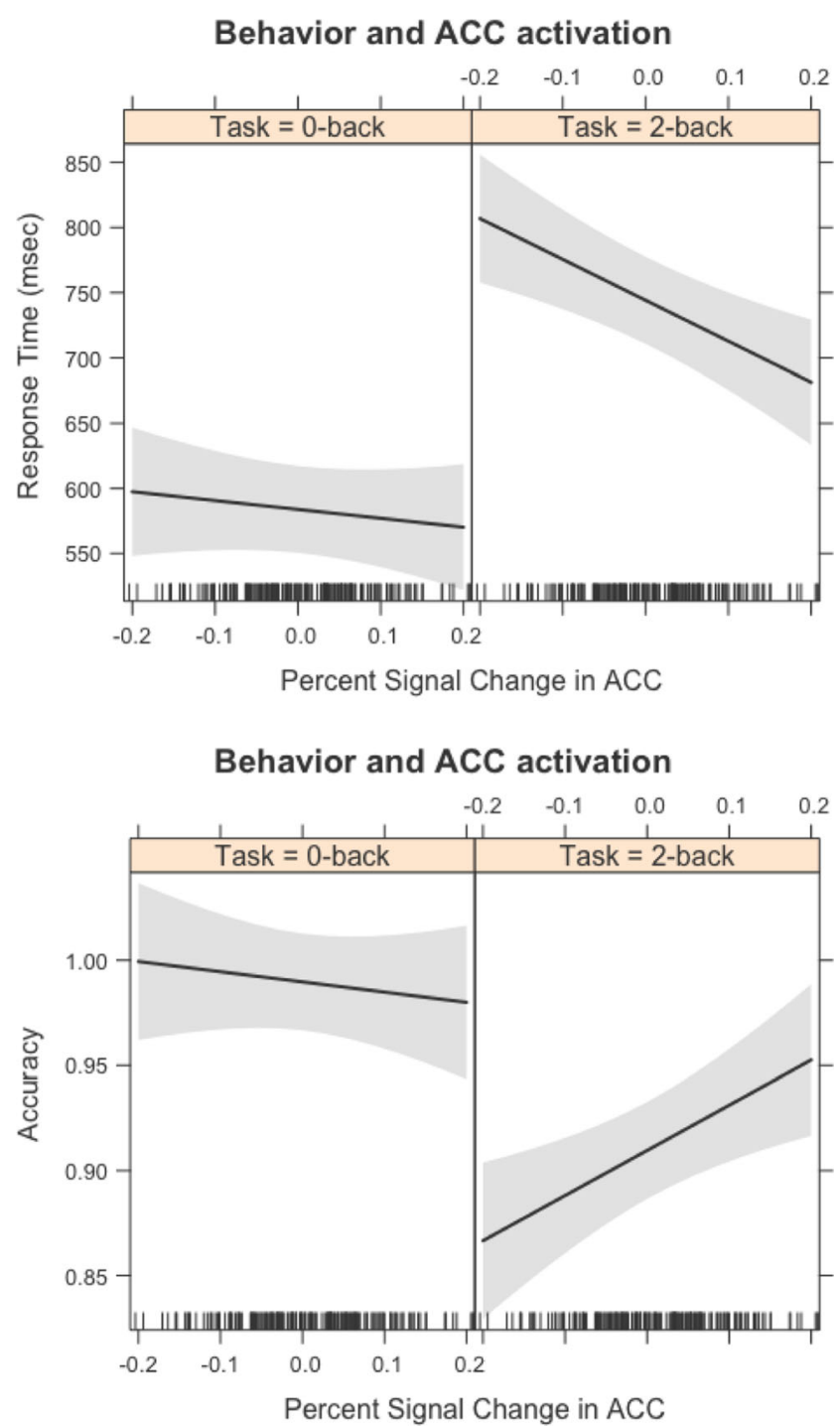

Fig. 4 Interaction between behavioral responses and activation in the anterior cingulate cortex (ACC). For both RTs (top) and accuracy (bottom) there was a significant interaction between task and brain activation in the ACC

remained unchanged, though the numbers of voxels in the two clusters of activation differed (ACC cluster, 128 voxels; cingulate cluster, 73 voxels).

Finally, to ensure that the association between brain activation and fatigue was specific to fatigue (and was not, e.g., general to any sort of self-reported state), we reran the Fatigue_average analysis including the average scores for each of the VASs that were administered between each run of the tasks (fatigue, happiness, sadness, anger, tension, and pain). Including these other self-report measures did not change the results associated with fatigue, though the numbers of voxels in the two clusters of activation once again differed (ACC cluster, 111 voxels; cingulate cluster, 116 voxels). This suggests that the results reported here are indeed specific to fatigue. 


\section{Discussion}

In the present study, we examined whether the same regions of the brain are activated during error processing as are activated in response to increasing cognitive fatigue. We expected this overlap based on theoretical considerations, as well as on separate empirical reports in the literatures on error processing and cognitive fatigue. Our results are clear: Areas that are activated by error processing are also activated when subjects experience fatigue. Although we did not anticipate the interaction between fatigue and task, which is discussed more below, these results represent the first formal test of the idea that outcome prediction and cognitive fatigue rely on partially overlapping neural networks.

Our results are in line with the prediction of response outcome (PRO) model of cognitive control (Alexander \& Brown, 2010), as well as other prediction error models such as the Rescorla-Wagner and temporal-difference models (Joel, Niv, \& Ruppin, 2002), which propose that the ACC has a role in the comparison of expected results with actual outcomes. Not only has the ACC been found to be important in outcome prediction in humans, but animal research has also shown this region to be involved in processing effort-related information (e.g., Engström et al. 2015; Holec, Pirot, \& Euston, 2014; Salamone, Correa, Nunes, Randall, \& Pardo, 2012; Walton, Bannerman, Alterescu, \& Rushworth, 2003).

One implication of this work is that the fatigue we experience may be an important part of the cognitive control system. Indeed, the feeling of fatigue may be one way that the brain signals itself that the payoff matrix has changed and that the reward being received no longer merits the effort being expended - an important aspect of self-regulation. This idea is analogous to the hypothesis in the literature on physical fatigue that fatigue is an emotion, produced by the brain during exercise, to ensure that physical homeostasis is not compromised by physical exertion (Noakes, 2012).

Another implication of the present study derives from the fact that in the present work, in which only healthy individuals were included, we found fatigue-related activation in the same ACC areas that are often found to be related to fatigue in individuals with brain injury or disease (Kohl et al., 2009; Roelcke et al., 1997). This suggests that the mechanisms of fatigue may operate similarly in healthy individuals and in clinical populations, and that the higher levels of fatigue reported by individuals with brain injury and disease may derive from a chronic disruption of the payoff matrix. For example, if a brain injury/disease results in inefficiency in information processing, then the effort required to perform many cognitive tasks would be expected to increase. The rewards individuals can expect to receive for this cognitive work, however, do not increase after brain injury/disease, and this results in a chronic imbalance between effort expended and reward received. Indeed, a separate line of work suggests that effort leads to a discounting of the value of outcomes, such that individuals value those outcomes more that follow less effortful actions (Botvinick, Huffstetler, \& McGuire, 2009; Scholl et al., 2015): Such discounting might exacerbate this chronic imbalance between effort and reward.

The idea that fatigue is an aspect of the cognitive control system is further supported by the behavioral data in the present study. As subjects' average fatigue increased, they responded with longer latencies and, during the two-back task, with increased accuracy. That is, increased average fatigue was associated with a speed-accuracy trade-off. In the signal detection literature, changes in the speed and accuracy of subjects' responses are understood to reflect a shift in bias ( $\beta$; Green \& Swets, 1966; Stanislaw \& Todorov, 1999). When subjects are given an incentive to respond more quickly, regardless of their accuracy, their latencies decrease, and their accuracy suffers. Alternatively, when subjects are given an incentive to respond accurately, regardless of speed, their latencies increase, and their accuracy improves. In both of these examples, changes in the payoff matrix, or the incentive structure, that the experimenter uses result in changes in $\beta$. In the present experiment, we propose that as the experiment progressed, subjects began to deplete their cognitive resources, and continued task performance then required increased effort. However, although the amount of effort that subjects had to expend to achieve adequate task performance increased, the reward that subjects derived from continued task performance did not. Put another way, there was a gradual shift in the payoff matrix. Given the signal detection literature, one would expect such a shift to result in a change in $\beta$, which would be detectable as a shift in the relative weight that subjects gave to responding quickly versus responding accurately. This shift is exactly what we observed: To the extent that subjects reported more average fatigue, they traded accuracy for speed. This result further supports the idea that fatigue is related to the balance between effort and reward.

A further issue involves the interrelationship between fatigue, task engagement, effort, and motivation. When subjects were performing the more difficult two-back task, it is intuitive to suppose that they were more engaged and that they expended more effort than during the less difficult zero-back task. Indeed, because the two-back task is more challenging, it may be intrinsically more motivating (or require more motivation to complete) than the zero-back task (Ryan \& Deci, 2000). Given this, one might expect that the ACC would be more active during the two-back task than during the zeroback task. This is because the ACC, and the larger dopaminergic reward network, is sensitive to motivation, becoming more activated when subjects are more motivated and less activated when they are less motivated (Stoppel et al., 2011; Vassena et al., 2014). In our results, when activation was compared across the two tasks, we found more activation in the ACC during the two-back task than during the zero-back task 
(see Supplemental Fig. 5). Furthermore, when we analyzed the relationship between activation in the ACC and behavioral performance, we found that increased activation during the two-back task was associated with better performance (in terms of both shorter RTs and higher accuracy). Thus, there is reason to suppose that the regions of the ACC identified here are sensitive to the motivational states of subjects, with more activation being associated with higher motivation, which is also related to better performance.

This conclusion is further bolstered by the analyses in which the fatigue that subjects reported before each block ("Fatigue_pre") and after each block ("Fatigue_post") were entered separately into the model. The analyses of RTs showed that when subjects reported more fatigue before a block of the zero-back task, they responded with longer latencies on the zero-back task. This might suggest that during the zero-back task, self-reported fatigue might be influenced by task engagement: When subjects are less engaged, they report more fatigue, and subsequently respond with longer RTs. This was not the case for the two-back task: On that task, when subjects responded with longer latencies, they reported more fatigue after the task. This is entirely compatible with the idea that subjects expended more effort on the two-back task, since longer latencies on that task are associated with more fatigue following task performance. This interpretation is bolstered by the BOLD data: More activation in the cingulate during the two-back task was associated with more fatigue after completion of that task, a relationship not evident in the data from the zero-back task.

Moreover, the idea that the same regions of the cingulate and ACC that are sensitive to motivation are also sensitive to fatigue was further supported by the findings from the secondary analyses we undertook to rule out alternative explanations. In those secondary analyses, we showed that the fatigue-related activation remained even after several potential metrics of task engagement were covaried out of the data. In our analyses of average fatigue and Fatigue post, as well as in our secondary analyses, brain activation in the ACC regions showed a positive correlation with fatigue during the two-back task and a negative correlation with fatigue during the zero-back task. That is, during the two-back task - which was more difficult, more intrinsically motivating, and more effortful - increasing activation in the ACC was associated with increasing fatigue; during the zero-back task - which was less difficult, less intrinsically motivating, and less effortful - increasing deactivation of the ACC was associated with increasing fatigue. It is not clear why the ACC responds in this way to the differential task demands (see Lim et al., 2010, for a related result), but these data do clearly show that that ACC activation is related to fatigue. Future work should manipulate motivation and effort to better understand these task-related effects.

Taken together, these results suggest that although ACC and cingulate regions are sensitive to subjects' motivational state (i.e., to task engagement), these regions are also sensitive to subjects' experience of fatigue. Given our hypothesis that fatigue represents a change in the balance between effort and reward, it is perhaps not surprising that brain areas sensitive to motivation (which is itself closely related to both effort and reward) are also associated with fatigue. An important avenue of future work will be to disentangle the interrelated concepts of fatigue, task engagement, effort, and motivation.

One limitation of the present study was that subjects were not given feedback, and it is therefore possible that they were not aware of all the errors they committed. Despite this possibility, robust activation in ACC was associated with error trials, which is not surprising, given that the ACC has been shown to be equally active when subjects are aware of the errors they commit and when they are not (Hester et al., 2005). Nevertheless, future work might benefit from providing subjects with feedback. It may also be valuable to investigate whether interleaving the zero-back and two-back blocks would affect subjects' fatigue. Here, subjects worked through a series of blocks of each task, which was done because we expected that blocking task difficulty in this way would amplify differences in fatigue. However, interleaving the tasks would provide better control of order effects and might also minimize effects of task engagement. Future work should also investigate the role of the ACC in clinical populations in which cognitive fatigue is known to be a problem (e.g., individuals with traumatic brain injury, Parkinson's disease, stroke, or multiple sclerosis). If cognitive fatigue in these populations results, at least in part, from a chronic shift in the payoff matrix, one would predict that the ACC would be chronically active. The effect of this on the rest of the cognitive system, and on the cognitive-control network in particular, is an important avenue of future research.

\section{Conclusion}

Here we presented evidence supporting the idea that cognitive fatigue is related to changes in effort and reward that result from changes in the payoff matrix. We speculate that as the brain detects these changes in the payoff matrix, cognitive fatigue is generated as a way for the brain to signal itself that the effort required for the task no longer merits the rewards received for performing it. This line of thinking is compatible with the idea that part of the network of areas associated with fatigue should include the ACC, inasmuch as the ACC has been shown to be sensitive to outcome predictions and error detection. The data presented here support this idea, showing that the same voxels in the ACC are sensitive to error processing and to cognitive fatigue. 
Author note The authors acknowledge grant support from the New Jersey Commission for Brain Injury Research (10.005.BIR1 to G.W. and H.G.), from the National Multiple Sclerosis Society (RG 4232A1 to H.G. and G.W.), and from the Kessler Foundation.

\section{References}

Alexander, W. H., \& Brown, J. W. (2010). Computational models of performance monitoring and cognitive control. Topics in Cognitive Science, 2, 658-677.

Boksem, M. A. S., Meijman, T. F., \& Lorist, M. M. (2006). Mental fatigue, motivation and action monitoring. Biological Psychology, 72, 123-132.

Boksem, M. A. S., \& Tops, M. (2008). Mental fatigue: Costs and benefits. Brain Research Reviews, 59, 125-139.

Botvinick, M. M., Huffstetler, S., \& McGuire, J. T. (2009). Effort discounting in human nucleus accumbens. Cognitive, Affective, \& Behavioral Neuroscience, 9, 16-27.

Botvinick, M., Nystrom, L. E., Fissell, K., Carter, C. S., \& Cohen, J. D. (1999). Conflict monitoring versus selection-for-action in anterior cingulate cortex. Nature, 402, 179-181. doi:10.1038/46035

Brown, J. W., \& Braver, T. S. (2005). Learned predictions of error likelihood in the anterior cingulate cortex. Science, 307, 1118-1121. doi:10.1126/science. 1105783

Carp, J., Fitzgerald, K. D., Taylor, S. F., \& Weissman, D. H. (2012). Removing the effect of response time on brain activity reveals developmental differences in conflict processing in the posterior medial prefrontal cortex. NeuroImage, 59, 853-860.

Chaudhuri, A., \& Behan, P. O. (2000). Fatigue and basal ganglia. Journal of the Neurological Sciences, 179, 34-42.

Cox, R. W. (1996). AFNI: Software for analysis and visualization of functional magnetic resonance neuroimages. Computers and Biomedical Research, 29, 162-173.

DeLuca, J., Genova, H. M., Hillary, F. G., \& Wylie, G. (2008). Neural correlates of cognitive fatigue in multiple sclerosis using functional MRI. Journal of Neurological Science, 270, 28-39.

Dobryakova, E., DeLuca, J., Genova, H. M., \& Wylie, G. R. (2013). Neural correlates of cognitive fatigue: Cortico-striatal circuitry and effort-reward imbalance. Journal of the International Neuropsychological Society, 19, 1-5.

Dobryakova, E., Genova, H. M., DeLuca, J., \& Wylie, G. R. (2015). The dopamine imbalance hypothesis of fatigue in multiple sclerosis and other neurological disorders. Frontiers in Neurology, 6.

Engström, M., Karlsson, T., Landtblom, A.-M., \& Craig, A. D. B. (2015). Evidence of conjoint activation of the anterior insular and cingulate cortices during effortful tasks. Frontiers in Human Neuroscience, 8(1071), 1-12. doi:10.3389/fnhum.2014.01071

Genova, H. M., Rajagopalan, V., Deluca, J., Das, A., Binder, A., Arjunan, A., \& Wylie, G. (2013). Examination of cognitive fatigue in multiple sclerosis using functional magnetic resonance imaging and diffusion tensor imaging. PLoS ONE, 8(e78811), 1-10. doi:10.1371/journal.pone.0078811

Green, D. G., \& Swets, J. A. (1966). Signal detection theory and psychophysics. New York: Wiley.

Grinband, J., Savitskaya, J., Wager, T. D., Teichert, T., Ferrera, V. P., \& Hirsch, J. (2011). The dorsal medial frontal cortex is sensitive to time on task, not response conflict or error likelihood. NeuroImage, 57, 303-311.

Hester, R., Foxe, J. J., Molholm, S., Shpaner, M., \& Garavan, H. (2005). Neural mechanisms involved in error processing: A comparison of errors made with and without awareness. NeuroImage, 27, 602-608.

Holec, V., Pirot, H. L., \& Euston, D. R. (2014). Not all effort is equal: The role of the anterior cingulate cortex in different forms of effort- reward decisions. Frontiers in Behavioral Neuroscience, 8(12), 117. doi:10.3389/fnbeh.2014.00012

Joel, D., Niv, Y., \& Ruppin, E. (2002). Actor-critic models of the basal ganglia: New anatomical and computational perspectives. Neural Networks, 15, 535-547.

Kohl, A. D., Wylie, G. R., Genova, H. M., Hillary, F. G., \& Deluca, J. (2009). The neural correlates of cognitive fatigue in traumatic brain injury using functional MRI. Brain Injury, 23, 420-432.

Lim, J., Wu, W., Wang, J., Detre, J. A., Dinges, D. F., \& Rao, H. (2010). Imaging brain fatigue from sustained mental workload: An ASL perfusion study of the time-on-task effect. NeuroImage, 49, 3426-3435.

Lorist, M. M., Bezdan, E., ten Caat, M., Span, M. M., Roerdink, J. B. T. M., \& Maurits, N. M. (2009). The influence of mental fatigue and motivation on neural network dynamics: An EEG coherence study. Brain Research, 1270, 95-106.

Mohamed, M. A., Yousem, D. M., Tekes, A., Browner, N., \& Calhoun, V. D. (2004). Correlation between the amplitude of cortical activation and reaction time: A functional MRI study. American Journal of Roentgenology, 183, 759-765.

Niv, Y., \& Schoenbaum, G. (2008). Dialogues on prediction errors. Trends in Cognitive Sciences, 265-272. doi:10.1016/j.tics.2008.03.006.

Noakes, T. D. (2012). Fatigue is a brain-derived emotion that regulates the exercise behavior to ensure the protection of whole body homeostasis. Frontiers in Physiology, 3(82), 113. doi: $10.3389 /$ fphys.2012.00082

Pardini, M., Bonzano, L., Mancardi, G. L., \& Roccatagliata, L. (2010). Frontal networks play a role in fatigue perception in multiple sclerosis. Behavioral Neuroscience, 124, 329-336.

Pardini, M., Krueger, F., Raymont, V., \& Grafman, J. (2010). Ventromedial prefrontal cortex modulates fatigue after penetrating traumatic brain injury. Neurology, 74, 749-754.

Roelcke, U., Kappos, L., Lechner-Scott, J., Brunnschweiler, H., Huber, S., Ammann, W., \& Leenders, K. L. (1997). Reduced glucose metabolism in the frontal cortex and basal ganglia of multiple sclerosis patients with fatigue: A $18 \mathrm{~F}$-fluorodeoxyglucose positron emission tomography study. Neurology, 48, 1566-1571.

Rothengatter, T., \& Huguenin, R. D. (2004). Traffic and transport psychology: Theory and application. Proceedings of the ICTTP 2000. Amsterdam: Elsevier Science.

Rozand, V., Lebon, F., Papaxanthis, C., \& Lepers, R. (2015). Effect of mental fatigue on speed-accuracy trade-off. Neuroscience, 297, 219-230.

Ryan, R. M., \& Deci, E. L. (2000). Self-determination theory and the facilitation of intrinsic motivation, social development, and well-being. American Psychologist, 55, 68-78. doi:10.1037/0003-066X.55.1.68

Salamone, J. D., Correa, M., Nunes, E. J., Randall, P. A., \& Pardo, M. (2012). The behavioral pharmacology of effort-related choice behavior: Dopamine, adenosine and beyond. Journal of the Experimental Analysis of Behavior, 97, 125-146.

Schneider, W., Eschman, A., \& Zuccolotto, A. (2002). E-Prime user's guide. Pittsburgh: Psychology Software Tools, Inc.

Scholl, J., Kolling, N., Nelissen, N., Wittmann, M. K., Harmer, C. J., \& Rushworth, M. F. S. (2015). The good, the bad, and the irrelevant: Neural mechanisms of learning real and hypothetical rewards and effort. Journal of Neuroscience, 35, 11233-11251. doi:10.1523/JNEUROSCI.0396-15.2015

Schultz, W. (2016). Dopamine reward prediction-error signalling: A twocomponent response. Nature Reviews Neuroscience, 17, 183-195.

Stanislaw, H., \& Todorov, N. (1999). Calculation of signal detection theory measures. Behavior Research Methods, Instruments, \& Computers, 31, 137-149. doi:10.3758/BF03207704

Stoppel, C. M., Boehler, C. N., Strumpf, H., Heinze, H.-J. J., Hopf, J.-M. M., \& Schoenfeld, M. A. (2011). Neural processing of reward magnitude under varying attentional demands. Brain Research, 1383, 218-229. 
Talairach, J., \& Tournoux, P. (1988). Co-planar stereotaxic atlas of the human brain: 3-dimensional proportional system. An approach to cerebral imaging. Stuttgart: Thieme.

Vassena, E., Silvetti, M., Boehler, C. N., Achten, E., Fias, W., \& Verguts, T. (2014). Overlapping neural systems represent cognitive effort and reward anticipation. PLOS ONE, 9, e91008. doi:10.1371/journal.pone.0091008

Walton, M. E., Bannerman, D. M., Alterescu, K., \& Rushworth, M. F. S. (2003). Functional specialization within medial frontal cortex of the anterior cingulate for evaluating effort-related decisions. Journal of Neuroscience, 23, 6475-6479.

Yeung, N., Botvinick, M. M., \& Cohen, J. D. (2004). The neural basis of error detection: Conflict monitoring and the error-related negativity. Psychological Review, 111, 931-959. doi:10.1037/0033-295X.111.4.931

Zarr, N., \& Brown, J. W. (2016). Hierarchical error representation in medial prefrontal cortex. NeuroImage, 124, 238-247. 